\title{
An analytical derivation of the survival probability of muon penetrating through matters
}

\author{
Atsushi lyono, ${ }^{a}{ }^{*}$ S. Yamamoto, ${ }^{a}$ S. Tsuji, ${ }^{b}$ K. Okei, ${ }^{b}$ H. Matsumoto ${ }^{b}$ and T. \\ Nakatsuka ${ }^{c}$
}

${ }^{a}$ Department of Fundamental Science, Okayama University of Science, Okayama 700-0005, Japan

${ }^{b}$ Department of Natural Sciences, Kawasaki Medical School, Kurashiki 701-0192, Japan

${ }^{c}$ Laboratory of Information Science, Okayama Shoka University, Okayama 700-8601, Japan

E-mail: iyono@das.ous.ac.jp

The survival probabilities of muon after penetrating through matters are evaluated analytically by solving the diffusion equation, taking account of not only bremsstrahlung but also positronelectron pair production and photonuclear interactions, together with ionization loss. Inaccuracy of probabilities derived by the ordinary saddle point method, reaching to $e / \sqrt{2 \pi} \simeq 1.08$, is corrected by applying the complementary-probability method. Accuracies of the results are discussed by comparing them with those derived by a Monte Carlo method.

$37^{\text {th }}$ International Cosmic Ray Conference (ICRC 2021)

July 12th - 23rd, 2021

Online - Berlin, Germany

\footnotetext{
${ }^{*}$ Presenter
} 


\section{Introduction}

We proposed in the preceding report [1] an evaluation of penetrating probability of charged particle in matters, taking account only of bremsstrahlung together with ionization loss. This time we advance the theory to take account also of positron-electron pair production and photonuclear interaction [2] to evaluate the survival probability of muon penetrating through matters, according to the same analytical flow as the preceding report.

We compare the results with those derived from our Monte Carlo simulation [3], where energy dissipations of muon are based on GEANT4 formulations [4] in main.

\section{The diffusion equation for energy dissipations of muon}

The diffusion equation for the differential energy spectrum $\pi\left(E_{0}, E, t\right)$ of charged particles, traversing through matters and dissipating their energies with fractional energy loss and ionization, is described as [1]

$$
\frac{\partial}{\partial t} \pi\left(E_{0}, E, t\right)=-\int_{0}^{1}\left\{\pi\left(E_{0}, E, t\right)-\frac{1}{1-v} \pi\left(E_{0}, \frac{E}{1-v}, t\right)\right\} \frac{\phi(v)}{b} d v+\varepsilon^{\prime} \frac{\partial}{\partial E} \pi\left(E_{0}, E, t\right),
$$

where $t$ denotes the traversed thickness measured in radiation unit, $\phi(v) d v$ the differential probability of fractional energy loss $v \equiv-\Delta E / E$ in each $\mathrm{g} / \mathrm{cm}^{2}$ of traversed thickness, $b^{-1}$ the radiation unit for muon expressed in $\mathrm{g} / \mathrm{cm}^{2}$, and $\varepsilon^{\prime}$ the constant energy loss in unit radiation length [5]. If we introduce M-function of $\mathcal{M}(s, q, t)$ by [6]

$$
\pi\left(E_{0}, E, t\right) d E=\frac{d E}{(2 \pi i)^{2}} \iint \frac{d s}{E}\left(\frac{E_{0}}{E}\right)^{s} \Gamma(-q)\left(\frac{\varepsilon^{\prime}}{E}\right)^{q} \mathcal{M}(s, q, t) d q,
$$

the diffusion equation (1) is described as

$$
\left\{\frac{\partial}{\partial t}+A(s+q)\right\} \mathcal{M}(s, q, t)=(s+q) q \mathcal{M}(s, q-1, t) \quad \text { with } \quad \mathcal{M}(s, 0,0)=1,
$$

where $A(s)$ denotes the characteristic energy-dissipating function derived by

$$
A(s) \equiv b^{-1} \int_{0}^{1}\left\{1-(1-v)^{s}\right\} \phi(v) d v .
$$

In case of the energy dissipations for muon, it satisfies $b \simeq 4.0 \times 10^{-6} \mathrm{~g}^{-1} \mathrm{~cm}^{2}$ and $\varepsilon^{\prime} \simeq 625$ $\mathrm{GeV}$ for Standard Rock $(Z=11, A=22)$ [5]. And as the probability density $\phi(v)$ for muon is additive as

$$
\phi(v) \equiv \phi_{\mathrm{P}}(v)+\phi_{\mathrm{B}}(v)+\phi_{\mathrm{N}}(v)
$$

among dissipating elements of positron-electron pair production (P), bremsstrahlung (B), and photonuclear interaction $(\mathrm{N})$ [2], the characteristic function $A(s)$ is also additive as

$$
A(s) \equiv A_{\mathrm{P}}(s)+A_{\mathrm{B}}(s)+A_{\mathrm{N}}(s) .
$$

Note that we express the characteristic function $A(s)$ for muon in power series, as indicated in the last report [2] as its Eq. (7) with the coefficients of its Table 1. 


\section{The survival probabilities of muon under Approx. A}

\subsection{The results ordinarily derived by the saddle point method}

Without ionization loss (Approx. A), it satisfies $\varepsilon^{\prime}=0$ thus the differential energy spectrum $\pi\left(E_{0}, E, t\right)$ for muon is derived as the residue at $q=0$ of Eq. (2), so that we have

$$
\begin{aligned}
\mathcal{M}(s, 0, t) & =e^{-A(s) t} \text { and } \\
\pi\left(E_{0}, E, t\right) d E & =\frac{d E}{2 \pi i} \int \frac{d s}{E}\left(\frac{E_{0}}{E}\right)^{s} e^{-A(s) t},
\end{aligned}
$$

where $A(s)$ of muon is expressed as Eq. (6). Then the survival probability $\Pi\left(E_{0}, E, t\right)$ of muons with their energies greater than $E$ is obtained as

$$
\Pi\left(E_{0}, E, t\right) \equiv \int_{E}^{\infty} \pi\left(E_{0}, E, t\right) d E=\frac{1}{2 \pi i} \int \frac{d s}{s}\left(\frac{E_{0}}{E}\right)^{s} e^{-A(s) t},
$$

where the path of integration is taken at $0<\mathcal{R}(s)$. We can evaluate the probability ordinarily by the saddle point method,

$$
\begin{gathered}
\Pi\left(E_{0}, E, t\right) \simeq\left(E_{0} / E\right)^{\bar{s}} e^{-A(\bar{s}) t} / \sqrt{2 \pi\left\{1-\bar{s}^{2} A^{\prime \prime}(\bar{s}) t\right\}}, \quad \text { with } \\
\ln \left(E_{0} / E\right) \simeq A^{\prime}(\bar{s}) t+1 / \bar{s} \text { and } 0<\bar{s} .
\end{gathered}
$$

The survival probabilities of muon versus $E_{0} / E$ so obtained at $t=1,2,3,4$, and 5 are indicated in the left panel of Fig, 1 (dot lines, from left to right). Also, those versus $t$ with $E_{0} / E=10^{2}, 10^{3}, 10^{4}, 10^{5}$, and $10^{6}$ are indicated in the right panel of Fig, 1 (dot lines, from left to right).

\subsection{The results derived via the complementary probability}

As mentioned in the preceding report [1], the ordinary results derived by the saddle point method give ill accurate limiting probability of $e / \sqrt{2 \pi} \simeq 1.08$ [7], exceeding 1 , to $\Pi\left(E_{0}, E, t\right)$ in Fig, 1 at $E_{0} / E \rightarrow \infty$ in the left panel and at $t \rightarrow 0$ in the right panel. To avoid this inaccuracy, we introduced the complementary probability $\Pi^{*}\left(E_{0}, E, t\right)$ defined by

$$
\Pi^{*}\left(E_{0}, E, t\right) \equiv-\frac{1}{2 \pi i} \int \frac{d s}{s}\left(\frac{E_{0}}{E}\right)^{s} e^{-A(s) t},
$$

by replacing the path of integration to $-1<\mathcal{R}(s)<0$ [1]. The residue of 1 at $s=0$ causes the complementary relation of

$$
\Pi\left(E_{0}, E, t\right)+\Pi^{*}\left(E_{0}, E, t\right)=1,
$$

so that the survival probability at the ill accurate regions can also be evaluated by the saddle point method, improving as

$$
\begin{gathered}
\Pi\left(E_{0}, E, t\right) \simeq 1-\left(E_{0} / E\right)^{\bar{s}} e^{-A(\bar{s}) t} / \sqrt{2 \pi\left\{1-\bar{s}^{2} A^{\prime \prime}(\bar{s}) t\right\}}, \quad \text { with } \\
\ln \left(E_{0} / E\right) \simeq A^{\prime}(\bar{s}) t+1 / \bar{s} \quad \text { and } \quad-1<\bar{s}<0 .
\end{gathered}
$$

The results are indicated in Fig, 1 (thick solid lines), which give the exact limiting values of 1 at $E_{0} / E \rightarrow \infty$ in the left panel and at $t \rightarrow 0$ in the right panel. 


\section{The survival probabilities of muon under Approx. B}

\subsection{Solution of the survival probabilities}

Taking account of ionization loss (Approx. B), the differential-difference equation (3) can be expressed as

$$
\{\lambda+A(s+q)\} \mathcal{L}(s, q, \lambda)=(s+q) q \mathcal{L}(s, q-1, \lambda)+\delta_{q, 0}
$$

by applying Laplace transform to $\mathcal{M}(s, q, t)$,

$$
\mathcal{L}(s, q, \lambda)=\int_{0}^{\infty} e^{-\lambda t} \mathcal{M}(s, q, t) d t
$$

as applied by Nishimura in his (A.5.9) [6]. Then we have

$$
\mathcal{L}(s, q, \lambda)=\frac{\Gamma(s+q+1) \Gamma(q+1)}{\Gamma(s+1)} \frac{1}{\lambda+A(s)} \lim _{m \rightarrow \infty}\{\lambda+A(s+m+1)\}^{-q} \prod_{j=1}^{m} \frac{\lambda+A(s+q+j)}{\lambda+A(s+j)},
$$

thus the survival probability $\Pi\left(E_{0}, 0, t\right)$ of muons at $t$ is derived by integrating Eq. (2) from $E$ to infinity and taking the limiting value at $E \rightarrow 0$, as

$$
\begin{aligned}
\Pi\left(E_{0}, 0, t\right) & \equiv \frac{1}{2 \pi i} \int\left(\frac{E_{0}}{\varepsilon^{\prime}}\right)^{s} \Gamma(s) \mathcal{M}(s,-s, t) d s \\
& =\frac{1}{(2 \pi i)^{2}} \iint\left(\frac{E_{0}}{\varepsilon^{\prime}}\right)^{s} e^{t \lambda} \Gamma(s) \mathcal{L}(s,-s, \lambda) d s d \lambda .
\end{aligned}
$$

\subsection{The solution in case of large $E_{0} / \varepsilon^{\prime}$}

The survival probability $\Pi\left(E_{0}, 0, t\right)$ expressed in Eq. (19) can be evaluated as

$$
\begin{gathered}
\Pi\left(E_{0}, 0, t\right) \simeq\left(E_{0} / \varepsilon^{\prime}\right)^{\bar{s}} \Gamma(\bar{s}) \mathcal{M}(\bar{s},-\bar{s}, t) / \sqrt{2 \pi\left(\partial^{2} / \partial s^{2}\right) \ln \{\Gamma(\bar{s}) \mathcal{M}(\bar{s},-\bar{s}, t)\}}, \quad \text { with } \\
\ln \left(E_{0} / \varepsilon^{\prime}\right)+(\partial / \partial s) \ln \{\Gamma(\bar{s}) \mathcal{M}(\bar{s},-\bar{s}, t)\}=0 \quad \text { and } 0<\bar{s}
\end{gathered}
$$

by applying the saddle point method ordinarily, where $\Gamma(s) \mathcal{M}(s,-s, t)$ is expressed as

$$
\begin{aligned}
& \Gamma(s) \mathcal{M}(s,-s, t)=\sum_{k=0}^{\infty} \Gamma(s) \mathcal{M}_{k}(s,-s) e^{-A(s+k) t} \quad \text { with } \\
& \Gamma(s) \mathcal{M}_{0}(s,-s)=-\Gamma(-s) \lim _{m \rightarrow \infty}\{A(s+m+1)-A(s)\}^{s} \prod_{j=1}^{m} \frac{A(j)-A(s)}{A(s+j)-A(s)} \text { and } \\
& \Gamma(s) \mathcal{M}_{k}(s,-s)=-\Gamma(-s) \frac{A(k)-A(s+k)}{A(s)-A(s+k)} \lim _{m \rightarrow \infty}\{A(s+m+1)-A(s+k)\}^{s} \prod_{j=1, j \neq k}^{m} \frac{A(j)-A(s+k)}{A(s+j)-A(s+k)},
\end{aligned}
$$

as indicated ever [6, 8]. Derivatives of $\Gamma(s) \mathcal{M}(s,-s, t)$ are expressed as

$$
\begin{aligned}
& \frac{\partial}{\partial s}\{\Gamma(s) \mathcal{M}(s,-s, t)\}= \sum_{k=0}^{\infty}\left[\frac{\partial}{\partial s}\left\{\Gamma(s) \mathcal{M}_{k}(s,-s)\right\}-A^{\prime}(s+k) t\left\{\Gamma(s) \mathcal{M}_{k}(s,-s)\right\}\right] e^{-A(s+k) t}, \\
& \frac{\partial^{2}}{\partial s^{2}}\{\Gamma(s) \mathcal{M}(s,-s, t)\}=\sum_{k=0}^{\infty}\left[\frac{\partial^{2}}{\partial s^{2}}\left\{\Gamma(s) \mathcal{M}_{k}(s,-s)\right\}-2 A^{\prime}(s+k) t \frac{\partial}{\partial s}\left\{\Gamma(s) \mathcal{M}_{k}(s,-s)\right\}\right. \\
&\left.+\left\{A^{\prime}(s+k)^{2} t^{2}-A^{\prime \prime}(s+k) t\right\}\left\{\Gamma(s) \mathcal{M}_{k}(s,-s)\right\}\right] e^{-A(s+k) t} .
\end{aligned}
$$


The survival probabilities versus $E_{0} / \varepsilon^{\prime}$ so obtained at $t=1,2,3,4$, and 5 are indicated in the right panel of Fig. 2 (dot lines, from top to bottom). We approximated the summation of Eq. (22) by the first term $(k=0)$, as applied in the preceding report [1]. Derivatives of $\Gamma(s) \mathcal{M}_{k}(s,-s)$ are evaluated numerically. This method is valid at $10 \lesssim E_{0} / \varepsilon^{\prime}$, showing satisfactory convergence.

Though, the evaluations by Eq. (20) obviously give a little larger results at regions of $E_{0} / \varepsilon^{\prime} \gg$ 1 , exceeding 1 , due to the inaccuracy of the ordinary saddle point method. We evaluate the complementary probability of

$$
\begin{aligned}
& \Pi^{*}\left(E_{0}, 0, t\right) \equiv-\frac{1}{2 \pi i} \int\left(\frac{E_{0}}{\varepsilon^{\prime}}\right)^{s} \Gamma(s) \mathcal{M}(s,-s, t) d s \\
& \simeq\left(E_{0} / \varepsilon^{\prime}\right)^{\bar{s}} \Gamma(\bar{s}) \mathcal{M}(\bar{s},-\bar{s}, t) / \sqrt{2 \pi\left(\partial^{2} / \partial s^{2}\right) \ln \{\Gamma(\bar{s}) \mathcal{M}(\bar{s},-\bar{s}, t)\}}, \quad \text { with } \\
& \ln \left(E_{0} / \varepsilon^{\prime}\right)+(\partial / \partial s) \ln \{\Gamma(\bar{s}) \mathcal{M}(\bar{s},-\bar{s}, t)\}=0 \quad \text { and } \quad-1<\bar{s}<0 \text {, }
\end{aligned}
$$

then $\Pi\left(E_{0}, 0, t\right) \equiv 1-\Pi^{*}\left(E_{0}, 0, t\right)$ obtained through the relation of Eq. (13) give more accurate results reaching to 1 at $E_{0} / \varepsilon^{\prime} \rightarrow \infty$, as indicated in the right panel of Fig. 2 (thin solid lines, at $t=1,2,3,4$, and 5 from top to bottom). Note that $\Pi\left(E_{0}, 0, t\right)$ derived here can also be applied to obtain the probability in case of small $E_{0} / \varepsilon^{\prime}$, discussed in the next subsection.

Also, the survival probabilities versus $t$ with $E_{0} / \varepsilon^{\prime}=10,10^{2}, 10^{3}, 10^{4}, 10^{5}$, and $10^{6}$ evaluated by Eq. (20) are indicated in the right panel of Fig, 3 (dot lines, from left to right). We approximated the summation of Eq. (22) by the first term $(k=0)$. Then we can effectively determine $t$ by

$$
A^{\prime}(\bar{s}) t=\ln \left(E_{0} / \varepsilon^{\prime}\right)+(\partial / \partial s) \ln \left\{\Gamma(\bar{s}) \mathcal{M}_{0}(\bar{s},-\bar{s})\right\} .
$$

This method is valid at $10 \lesssim E_{0} / \varepsilon^{\prime}$, showing satisfactory convergence. The results evaluated by the ordinary saddle point method of Eq. (20) give ill accurate probability, where we improved the probability $\Pi\left(E_{0}, 0, t\right)$ through the complementary probability by Eqs. (13), (27), and (29) with $-1<\bar{s}<0$ as indicated in the right panel of Fig, 2 (solid lines, from left to right).

We compare the results with those derived from our Monte Carlo simulation [3]. We confirm fairy good agreements between the two in the right panels of Figs. 2 and 3. Though, the right panel of Fig, 3 shows a little larger survival probabilities in the analytical results than those in the Monte Carlo results, which will be caused by the smaller ionization loss with the constant value of $\varepsilon^{\prime} \simeq$ $625 \mathrm{GeV}$ taken by the former, than the slowly increasing ionization loss with increase of energy $E$ taken by the latter due to applying the Bethe-Bloch cross-section.

\subsection{The solution in case of small $E_{0} / \varepsilon^{\prime}$}

Evaluation of the survival probability by Eq. (20) described in the last subsection can not be applied any more in case of small $E_{0} / \varepsilon^{\prime}$, due to divergence of the results. Thus we derive the survival probability of Eq. (19) by

$$
\begin{aligned}
\Pi\left(E_{0}, 0, t\right) & =\frac{1}{2 \pi i} \int e^{t \lambda} \Xi\left(E_{0} / \varepsilon^{\prime}, \lambda\right) d \lambda \\
& \simeq e^{t \bar{\lambda}} \Xi\left(E_{0} / \varepsilon^{\prime}, \bar{\lambda}\right) / \sqrt{2 \pi\left(\partial^{2} / \partial \lambda^{2}\right)\left\{\ln \Xi\left(E_{0} / \varepsilon^{\prime}, \bar{\lambda}\right)\right\}}, \quad \text { with } \\
t & \simeq-(\partial / \partial \lambda)\left\{\ln \Xi\left(E_{0} / \varepsilon^{\prime}, \bar{\lambda}\right)\right\} \quad \text { and }-\infty<\bar{\lambda}<\infty,
\end{aligned}
$$


where we introduce $\Xi\left(E_{0} / \varepsilon^{\prime}, \lambda\right)$ as

$$
\begin{aligned}
\Xi\left(E_{0} / \varepsilon^{\prime}, \lambda\right) & =\frac{1}{2 \pi i} \int\left(\frac{E_{0}}{\varepsilon^{\prime}}\right)^{s} \Gamma(s) \mathcal{L}(s,-s, \lambda) d s \\
& =-\frac{1}{2 \pi i} \int d s\left(\frac{E_{0}}{\varepsilon^{\prime}}\right)^{s} \frac{\Gamma(-s)}{\lambda+A(s)} \lim _{m \rightarrow \infty}\{\lambda+A(s+m+1)\}^{s} \prod_{j=1}^{m} \frac{\lambda+A(j)}{\lambda+A(s+j)},
\end{aligned}
$$

with the path of integration taken at $0<\mathcal{R}(s)<1$. As $\Gamma(-s)$ gives the residue of $-(-1)^{k} / k$ ! at $s=k$, we have

$$
\begin{aligned}
\Xi\left(E_{0} / \varepsilon^{\prime}, \lambda\right) & =-\sum_{k=1}^{\infty} \frac{1}{k !}\left(-\frac{E_{0}}{\varepsilon^{\prime}}\right)^{k} \frac{1}{\lambda+A(k)} \lim _{m \rightarrow \infty}\{\lambda+A(k+m+1)\}^{k} \prod_{j=1}^{m} \frac{\lambda+A(j)}{\lambda+A(k+j)} \\
& =\frac{E_{0}}{\varepsilon^{\prime}}-\sum_{k=2}^{\infty} \frac{1}{k !}\left(-\frac{E_{0}}{\varepsilon^{\prime}}\right)^{k} \prod_{j=1}^{k-1}(\lambda+A(j)),
\end{aligned}
$$

and have the derivatives of $\Xi\left(E_{0} / \varepsilon^{\prime}, \lambda\right)$ as

$$
\begin{aligned}
& \frac{\partial}{\partial \lambda} \Xi\left(E_{0} / \varepsilon^{\prime}, \lambda\right)=-\sum_{k=2}^{\infty} \frac{1}{k !}\left(-\frac{E_{0}}{\varepsilon^{\prime}}\right)^{k} \prod_{j=1}^{k-1}(\lambda+A(j)) \sum_{j=1}^{k-1} \frac{1}{\lambda+A(j)} \\
& \frac{\partial^{2}}{\partial \lambda^{2}} \Xi\left(E_{0} / \varepsilon^{\prime}, \lambda\right)=-\sum_{k=3}^{\infty} \frac{2}{k !}\left(-\frac{E_{0}}{\varepsilon^{\prime}}\right)^{k} \prod_{j=1}^{k-1}(\lambda+A(j)) \sum_{j=1}^{k-2} \frac{1}{\lambda+A(j)} \sum_{m=j+1}^{k-1} \frac{1}{\lambda+A(m)} .
\end{aligned}
$$

The survival probabilities versus $E_{0} / \varepsilon^{\prime}$ so obtained at $t=0.2,0.4,0.6,0.8$, and 1.0 are indicated in the left panel of Fig, 2 (dot lines, from left to right). This method was valid at $E_{0} / \varepsilon^{\prime} \lesssim 2$. Though the results soon exceed 1 at larger $E_{0} / \varepsilon^{\prime}$ regions, where we replaced the results by applying the complementary probabilities of Eq. (27) (thin solid lines at $t=0.2,0.4,0.6,0.8$, and 1.0).

The complementary probability of Eq. (30) gives

$$
\begin{aligned}
\Pi^{*}\left(E_{0}, 0, t\right) & \equiv \frac{1}{2 \pi i} \int e^{t \lambda} \Xi^{*}\left(E_{0} / \varepsilon^{\prime}, \lambda\right) d \lambda \\
& \simeq e^{t \bar{\lambda}} \Xi^{*}\left(E_{0} / \varepsilon^{\prime}, \bar{\lambda}\right) / \sqrt{2 \pi\left(\partial^{2} / \partial \lambda^{2}\right)\left\{\ln \Xi^{*}\left(E_{0} / \varepsilon^{\prime}, \bar{\lambda}\right)\right\}}, \quad \text { with } \\
t & \simeq-(\partial / \partial \lambda)\left\{\ln \Xi^{*}\left(E_{0} / \varepsilon^{\prime}, \bar{\lambda}\right)\right\} \text { and } 0<\bar{\lambda},
\end{aligned}
$$

where

$$
\Xi^{*}\left(E_{0} / \varepsilon^{\prime}, \lambda\right) \equiv-\frac{1}{2 \pi i} \int\left(\frac{E_{0}}{\varepsilon^{\prime}}\right)^{s} \Gamma(s) \mathcal{L}(s,-s, \lambda) d s=1 / \lambda-\Xi\left(E_{0} / \varepsilon^{\prime}, \lambda\right)
$$

Note that the residue of $1 / \lambda$ at $s=0$ was taken into account as the path of integration was moved to $\mathcal{R}(s)<0$. And we have

$$
\begin{aligned}
& (\partial / \partial \lambda) \Xi^{*}\left(E_{0} / \varepsilon^{\prime}, \lambda\right)=-1 / \lambda^{2}-(\partial / \partial \lambda) \Xi\left(E_{0} / \varepsilon^{\prime}, \lambda\right), \\
& \left(\partial^{2} / \partial \lambda^{2}\right) \Xi^{*}\left(E_{0} / \varepsilon^{\prime}, \lambda\right)=2 / \lambda^{3}-\left(\partial^{2} / \partial \lambda^{2}\right) \Xi\left(E_{0} / \varepsilon^{\prime}, \lambda\right) .
\end{aligned}
$$

The survival probability $\Pi\left(E_{0}, 0, t\right) \equiv 1-\Pi^{*}\left(E_{0}, 0, t\right)$ obtained here from Eq. (36) partially interpolate that from Eq. (27) and that from Eq. (30), as indicated in the left panel of Fig, 2 (thick solid lines at $t=0.2$ and 0.4 , from left to right). 
Also, the survival probabilities versus $t$ with $E_{0} / \varepsilon^{\prime}=0.1,0.2,0.5,1.0$, and 2.0 are evaluated via the complementary probability of Eq. (27) in case of small probability decrease from 1 (thin solid lines, from left to right), by Eq. (30) in case of large probability decrease from 1 (dot lines, from left to right), and via Eq. (36) in case of interpolating the above cases, as indicated in the left panel of Fig, 3 (thick solid lines for $E_{0} / \varepsilon^{\prime}=0.1,0.2$, and 0.5 , from left to right).

We compare the results with those derived from our Monte Carlo simulation. The former shows more rapid decreases of the survival probability at small traversed thickness than the latter as indicated in the left panels of Figs. 2 and 3, which will be caused by harder cross-sections for the fractional energy loss applied in the former with $E \rightarrow \infty$ assumed than that applied in the latter, especially by difference of the bremsstrahlungs applied as indicated in Fig. 2 of the last report [2].

\section{Conclusions and discussions}

Survival probabilities of muon penetrating through matters are evaluated analytically by taking account of bremsstrahlung, positron-electron pair production and photonuclear interactions, together with ionization loss. The complementary-probability method was effective to remove inaccuracy of the ordinary saddle point method to give probabilities exceeding 1.

Comparison of the analytical results obtained this time with those derived from our Monte Carlo method shows the both agree fairy well. Though, systematic quantitative differences between the two are found which will be caused by the difference of energy-dissipation model in continuous ionization loss and discrete fractional dissipations. It will be important to apply the both, making use of the respective advantages.

\section{Acknowledgments}

The authors are very indebted to Prof. Jun Nishimura for valuable discussions and advices.

\section{References}

[1] Iyono A, Yamamoto S, Tsuji S, Okei K, Matsumoto H and Nakatsuka T, Conference Papers of 35th International Cosmic Ray Conference, Busan PoS (ICRC, 2017)565, https://pos.sissa.it/301/565/pdf.

[2] Iyono A, Yamamoto S, Tsuji S, Okei K, Matsumoto H and Nakatsuka T, Conference Papers of 26th Extended European Cosmic Ray Symposium, Altai Journal of Physics: Conf. Series 1181(2019)012069.

[3] K. Okei and T. Nakatsuka, Proc. of 29th ICRC (Pune), 6(2005)197.

[4] GEANT4, a simulation toolkit, https://geant4.web.cern.ch/support

[5] T. Nakatsuka, K. Kobayakawa, and T. Kitamura, Proc. of 20th ICRC (Moscow), 6(1987)261.

[6] J. Nishimura, in Handbuch der Physik, Band 46, edited by S. Flügge (Springer, Berlin, 1967), Teil 2, p. 1.

[7] L. Eyges, Phys. Rev., 76(1949)264.

[8] K. Kobayakawa, Nuovo Cim., B47(1967)156. 

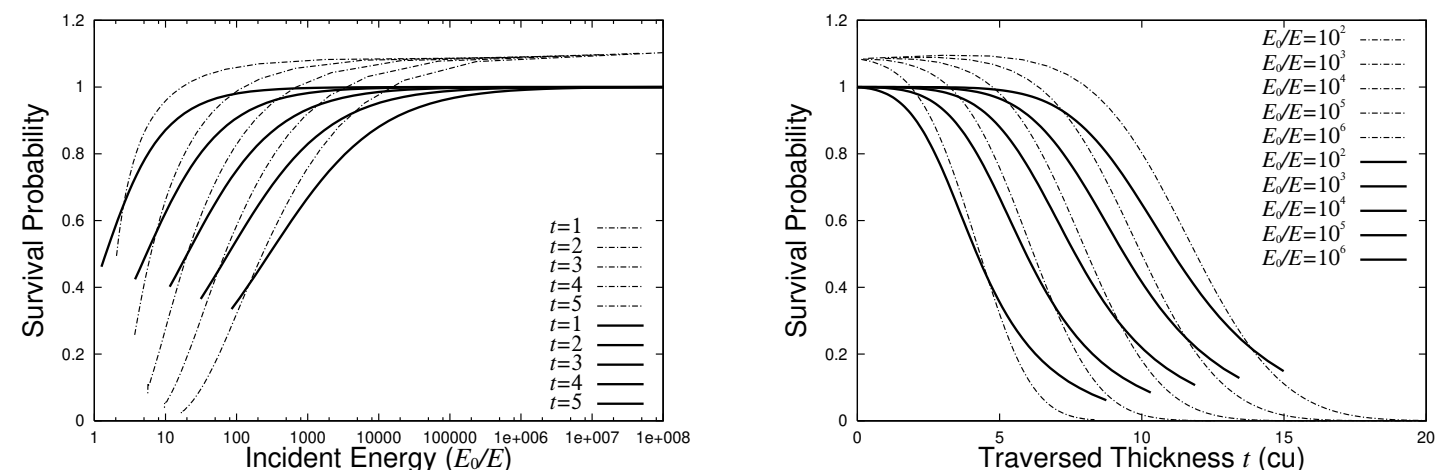

Figure 1: Survival probabilities with $\varepsilon^{\prime}=0$ (Approx. A) versus $E_{0} / E$ at $t=1,2,3,4,5$ (left panel) and those versus $t$ with $E_{0} / E=10^{2}, 10^{3}, 10^{4}, 10^{5}, 10^{6}$ (right panel), derived by the ordinary saddle point method (thin lines) and via the complementary-probability method (thick lines).
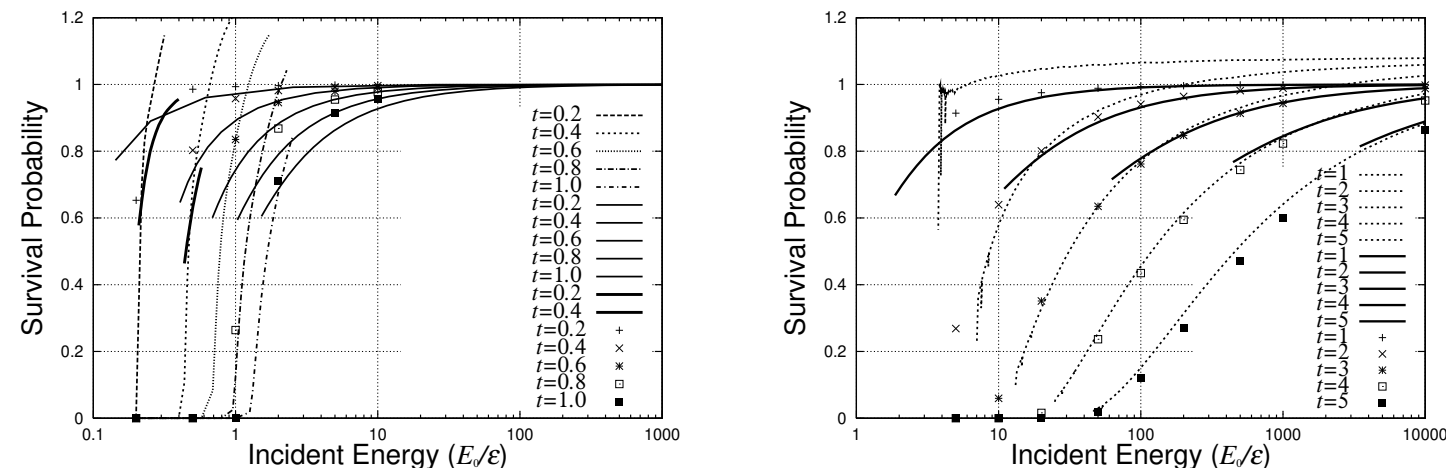

Figure 2: Survival probabilities (Approx. B) versus $E_{0} / \varepsilon^{\prime}$ at $t=0.2,0.4,0.6,0.8,1$ (left panel) and those at $t=1,2,3,4,5$ (right panel), derived by the ordinary saddle point method (thin dot lines), via the complementary-probability method (thick lines), via the extended complementary probabilities from large $E_{0} / \varepsilon^{\prime}$ region (thin lines in the left panel), and by Monte Carlo simulations (dots).
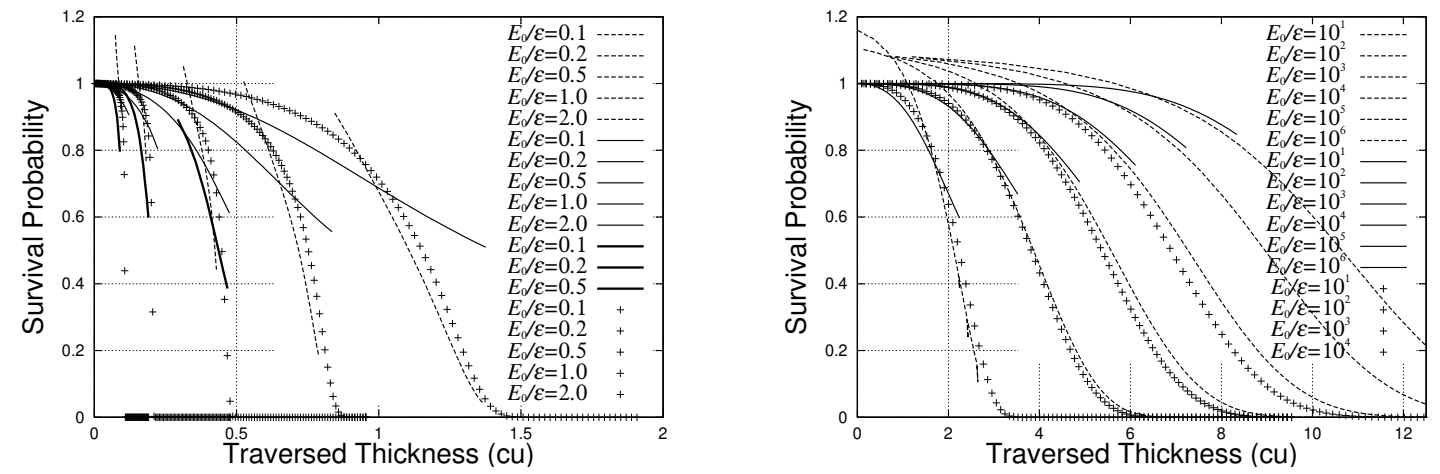

Figure 3: Survival probabilities (Approx. B) versus $t$ with $E_{0} / \varepsilon^{\prime}=0.10 .2,0.5,1.0,2.0$ (left panel) and those with $E_{0} / \varepsilon^{\prime}=10,10^{2}, 10^{3}, 10^{4}, 10^{5}, 10^{6}$ (right panel), derived by the ordinary saddle point method (thin lines), via the complementary-probability method (thick lines), and by Monte Carlo simulations (dots). 